\title{
Correlação Eletro-Ecocardiográfica no Diagnóstico da Hipertrofia Ventricular Esquerda
}

\author{
Hamilton Domingos, Josane Couto Elias Luzio, Gualberto Nogueira de Leles, Leandro Sauer, \\ Luiz Alberto Ovando
}

\author{
Campo Grande, MS
}

\begin{abstract}
Objetivo - Comparar a eficácia diagnóstica de 4 critérios eletrocardiográficos: índices de Sokolov, Gubner, Cornell e Romhilt, na deteç̧ão da hipertrofia ventricular esquerda (HVE) em pacientes hipertensos.

Métodos - Foram avaliados 30 pacientes ambulatoriais, com hipertensão arterial sistêmica leve, moderada ou grave, sendo considerado o diagnóstico de HVE quando os indices eram: Sokolov $\geq 35 \mathrm{~mm}$, Gubner $\geq 22 \mathrm{~mm}$, Romhilt $\geq 5$ pontos e Cornell $\geq 20 \mathrm{~mm}$ para mulheres $e$ 28 mm para homens. Determinaram-se, então, a sensibilidade, especificidade, eficácia diagnóstica e outras variáveis diagnósticas para cada um dos critérios, isoladamente, e para os 4 considerados simultaneamente, utilizandose como padrão ouro para HVE o indice de massa do ventrículo esquerdo $\geq 98 \mathrm{~g} / \mathrm{m}^{2}$ para mulheres e $\geq 120 \mathrm{~g} / \mathrm{m}^{2}$ para homens, obtido ao ecocardiograma.

Resultados - Considerando cada critério eletrocardiográfico isolado, o indice de Sokolov apresentou a melhor eficácia, com sensibilidade $=40 \%$, eficácia diagnóstica de $50 \%$ e especificidade $100 \%$; o $2^{\circ}$ critério mais eficaz foi o indice de Gubner e, em $3^{\circ}$ lugar, empatados, os indices de Romhilt e Cornell. Quando considerados, simultaneamente, ou seja, a presença de pelo menos um dos quatro critérios, a sensibilidade foi $52 \%$ e a eficácia diagnóstica 57\%.

Conclusão - Os quatro critérios eletrocardiográficos considerados individualmente ou em conjunto foram pouco eficazes na detecção da HVE e, considerando a importância desta entidade patológica, concluímos que é imprescindivel a utilização de método propedêutico mais sensivel para tal diagnóstico.
\end{abstract}

Palavras-chave: hipertrofia ventricular esquerda, eletrocardiografia, hipertensão

\section{Electro-Echocardiographic Correlation in the Diagnosis of Left Ventricular Hypertrophy}

Purpose - To compare the efficacy of four electrocardiographic criteria: Sokolov, Gubner, Cornell and Romhilt indexes, in the diagnosis of left ventricular hypertrophy (LVH) in hypertensive patients.

Methods - LVH was analyzed in the eletrocardiogram of 30 ambulatory patients presenting with systemic arterial hypertension, classified as mild, moderate and severe, according to the following indexes: Sokolov $\geq 35 \mathrm{~mm}$, Gubner $\geq 22 \mathrm{~mm}$, Romhilt $\geq 5$ points and Cornell $\geq 20 \mathrm{~mm}$ for women and $28 \mathrm{~mm}$ for men. Sensitivity, specificity, diagnostic accuracy and other diagnostic variables were determined. Mass index of the left ventricle, $\geq 98 \mathrm{~g} / \mathrm{m}^{2}$ for women and $\geq 120 \mathrm{~g} / \mathrm{m}^{2}$ for men, obtained by echocardiography, was considered the gold standard for the diagnosis of $L V H$.

Results - When electrocardiographic criteria were considered separately, the Sokolov index showed the highest accuracy, with a sensitivity of $40 \%$, diagnostic accuracy of $50 \%$ and specificity of $100 \%$; the second most accurate index was Gubner, and Romhilt and Cornell indexes followed. When at least one of the indexes was positive, the sensitivity was $52 \%$ and diagnostic accuracy was $57 \%$.

Conclusion - The four electrocardiographic indexes were not diagnostic of $L V H$, when analyzed either separately or together. Considering the high prevalence of this pathological condition, we conclude that a more accurate diagnostic method should be used in this diagnosis.

Key-words: left ventricular hypertrophy, electrocardiography, hypertension

Arq Bras Cardiol, volume 71 (n 1), 31-35, 1998

Universidade Federal de Mato Grosso do Sul - Campo Grande

Correspondência: Hamilton Domingos - Rua Dezoito de Setembro, 86 - 79005-200 - Campo Grande, MS

Recebido para publicação em 30/6/97

Aceito em 20/1/98
A associação entre hipertensão arterial sistêmica (HAS) e hipertrofia ventricular esquerda (HVE) é bem conhecida, tendo sido evidenciada no estudo de Framinghan, com base nos dados de prevalência encontrados. Dos 5.209 pacientes avaliados nos primeiros 12 anos, $76(1,5 \%)$ apre- 
sentavam HVE definida ao eletrocardiograma (ECG) e 88 $(1,7 \%)$ apresentavam possível HVE. Nessa mesma população, a prevalência de HVE ao ecocardiograma foi mais comum em homens do que em mulheres, acometendo $2 \%$ na faixa etária de $49-54$ anos e $10 \%$ entre 75 e 80 anos ${ }^{1}$.

A HVEé um fator de risco independente de morbimortalidade cardiovascular ${ }^{2}$, sendo portanto, lógica a importância de sua detecção precoce, bem como a adoção de medidas terapêuticas visando a sua regressão.

Na literatura são indicadas sensibilidade e especificidade divergentes em relação à HVE ao ECG, causando certa insegurança na utilização apenas do critério eletrocardiográfico na exclusão da HVE. Vários critérios têm sido propostos, mas, infelizmente, aqueles com alta especificidade (comprovada por necropsia) têm uma sensibilidade relativamente baixa ${ }^{1}$. Critérios de ECG tradicionais, baseados na combinação de voltagens, têm baixa sensibilidade para HVE e, geralmente, falham na identificação de pacientes com aumento leve a moderado da massa ventricular ${ }^{3}$.

Embora o ecocardiograma possa medir com acurácia a massa do ventrículo esquerdo (VE), seu uso, em grande parte da população, é limitado pelo custo, pela baixa disponibilidade do método e por dificuldade técnica ocasional ${ }^{4}$. Quando háHVEaoECG, existe jáao ecocardiograma aumento da massa ventricular em quase $100 \%$ dos casos, enquanto que em menos de $50 \%$ deles encontra-se dilatação ventricular ${ }^{5}$.
Neste trabalho, objetivando a determinação da sensibilidade e da eficiência de quatro critérios eletrocardiográficos na detecção da HVE, correlacionando-os com a massa ventricular esquerda obtida ao ecocardiograma, num grupo de 30 pacientes hipertensos em acompanhamento ambulatorial.

\section{Métodos}

Foram estudados, prospectivamente, 30 adultos hipertensos do Ambulatório do Serviço de Cardiologia do Hospital Universitário da UFMS, analisados os traçados eletrocardiográficos, a fim de determinar cada um dos quatro critérios e realizados os ecocardiogramas para determinação da massa do VE.

A amostra incluiu pacientes hipertensos com HAS leve, moderada ou grave, utilizando-se o $5^{\circ}$ ruído de Korotkoff para a diástole. Excluíram-se os portadores de insuficiência cardíaca congestiva (fração de ejeção <50\%), os portadores de valvulopatias, os coronariopatas (infarto agudo do miocárdio prévio), os nefropatas em diálise e os hipertensos sistólicos.

Dos 30 pacientes avaliados, $12(40 \%)$ eram homens e 18 mulheres, $15(50 \%)$ pardos, $13(43 \%)$ brancos e dois $(7 \%)$ negros. As idades variaram de 26 a 78 anos, sendo quatro (14\%) pacientes entre 26 e 39 anos, 13 (43\%) entre 40 e 60 anos e $13(43 \%)$ entre 61 e 78 anos. Quanto aos níveis

\begin{tabular}{|c|c|c|c|c|c|c|c|c|}
\hline \multicolumn{9}{|c|}{ Quadro I - Índices eletrocardiográficos } \\
\hline $\mathrm{N}^{\mathrm{O}}$ & Idade & Sexo & Cor & $\begin{array}{l}\text { Grau } \\
\text { HAS }\end{array}$ & $\begin{array}{l}\text { Sokolov } \\
\text { mm }\end{array}$ & $\begin{array}{c}\text { Romhilt } \\
\text { pontos }\end{array}$ & $\begin{array}{c}\text { Cornell } \\
\text { mm }\end{array}$ & $\begin{array}{c}\text { Gubner } \\
\mathrm{mm}\end{array}$ \\
\hline 1 & 66 & M & $\mathrm{P}$ & $\mathrm{G}$ & 30 & 3 & 20 & 16 \\
\hline 2 & 61 & $\mathrm{~F}$ & B & M & 25 & 0 & 6 & 11 \\
\hline 3 & 33 & $\mathrm{M}$ & $\mathrm{P}$ & $\mathrm{L}$ & 28 & 1 & 12 & 7 \\
\hline 4 & 59 & M & B & $\mathrm{L}$ & 26 & 3 & 7 & 12 \\
\hline 5 & 13 & M & $\mathrm{P}$ & $\mathrm{G}$ & 38 & 1 & 21 & 19 \\
\hline 6 & 58 & $\mathrm{~F}$ & B & M & 17 & 0 & 15 & 18 \\
\hline 7 & 70 & $\mathrm{~F}$ & $\mathrm{P}$ & $\mathrm{M}$ & 40 & 4 & 15 & 17 \\
\hline 8 & 76 & $\mathrm{~F}$ & $\mathrm{P}$ & $\mathrm{L}$ & 40 & 1 & 23 & 23 \\
\hline 9 & 64 & $\mathrm{~F}$ & $\mathrm{~N}$ & $\mathrm{~L}$ & 25 & 3 & 13 & 10 \\
\hline 10 & 44 & $\mathrm{~F}$ & B & M & 23 & 0 & 8 & 7 \\
\hline 11 & 35 & M & $\mathrm{P}$ & $\mathrm{G}$ & 13 & 1 & 21 & 3 \\
\hline 12 & 60 & $\mathrm{~F}$ & B & $\mathrm{L}$ & 12 & 1 & 12 & 13 \\
\hline 13 & 26 & M & $\mathrm{N}$ & M & 45 & 7 & 23 & 28 \\
\hline 14 & 68 & $\mathrm{~F}$ & B & $\mathrm{L}$ & 24 & 1 & 16 & 31 \\
\hline 15 & 72 & M & B & $\mathrm{L}$ & 30 & 0 & 11 & 13 \\
\hline 16 & 61 & $\mathrm{~F}$ & B & M & 35 & 1 & 17 & 18 \\
\hline 17 & 53 & $\mathrm{~F}$ & $\mathrm{P}$ & $\mathrm{L}$ & 20 & 0 & 12 & 17 \\
\hline 18 & 56 & $\mathrm{~F}$ & $\mathrm{P}$ & $\mathrm{G}$ & 47 & 6 & 10 & 13 \\
\hline 19 & 72 & $\mathrm{~F}$ & B & G & 15 & 0 & 6 & 8 \\
\hline 20 & 49 & M & $\mathrm{P}$ & G & 25 & 1 & 10 & 6 \\
\hline 21 & 53 & $\mathrm{~F}$ & B & M & 37 & 1 & 12 & 22 \\
\hline 22 & 70 & $\mathrm{~F}$ & B & G & 19 & 1 & 19 & 23 \\
\hline 23 & 60 & M & B & $\mathrm{G}$ & 28 & 5 & 31 & 11 \\
\hline 24 & 59 & M & $\mathrm{P}$ & M & 33 & 4 & 17 & 10 \\
\hline 25 & 53 & $\mathrm{~F}$ & $\mathrm{P}$ & M & 35 & 3 & 32 & 24 \\
\hline 26 & 43 & $\mathrm{~F}$ & $\mathrm{P}$ & $\mathrm{L}$ & 38 & 1 & 6 & 11 \\
\hline 27 & 46 & $\mathrm{~F}$ & $\mathrm{P}$ & $\mathrm{L}$ & 27 & 0 & 12 & 20 \\
\hline 28 & 37 & $\mathrm{~F}$ & $\mathrm{P}$ & $\mathrm{L}$ & 24 & 0 & 4 & 5 \\
\hline 29 & 78 & M & B & $\mathrm{L}$ & 18 & 0 & 7 & 7 \\
\hline 30 & 61 & $\mathrm{M}$ & $\mathrm{P}$ & $\mathrm{L}$ & 35 & 3 & 18 & 24 \\
\hline
\end{tabular}


tensionais, 13 (43\%) eram hipertensos leves, nove (30\%) moderados e oito (27\%) graves, considerando a classificação da $5^{\text {a }}$ Revisão procedida pelo Joint National Committee on Detection, Evaluation and Treatment of High Blood Pressure (EUA-1993) ${ }^{6}$.

Os seguintes critérios eletrocardiográficos foram selecionados para análise: 1) índice de Sokolov-Lyon ${ }^{4}$ : onda $S$ de V1+ onda R de V5 ou V6 $\geq 35 \mathrm{~mm} ; 2$ ) índice de Romhilt ${ }^{4}$ (escore de pontos): $\geq 5$ pontos: a) onda $\mathrm{R}$ ou $\mathrm{S}$ periféricos $\geq 20 \mathrm{~mm}$, ou onda S de V1 ou V2 $\geq 30 \mathrm{~mm}$, ou onda R de V5 ou $\mathrm{V} 6 \geq 30 \mathrm{~mm}-3$ pontos; $b$ ) infradesnivelamento de $\mathrm{ST}+\mathrm{T}$ invertida (strain): sem digital -3 pontos; com digital - 1 ponto; c) aumento de átrio esquerdo - índice de Morris $\geq 0,04 \mathrm{~mm} \mathrm{X}$ s - 3 pontos; d) SÂQRS $\geq-30$ graus - 2 pontos; e) tempo de ativação ventricular $\geq 0,05 \mathrm{~s}-1$ ponto; f) duração do $\mathrm{QRS} \geq$ 0,09s - 1 ponto; 3 ) índice de Gubner ${ }^{7}$ : onda R de D1 + onda S deD3 $\geq 22 \mathrm{~mm}$; 4) índice de Cornell ${ }^{8}$ : onda R de aVL + onda $\mathrm{Sde}$ V $\geq 20 \mathrm{~mm}$ para mulheres e $28 \mathrm{~mm}$ para homens.

Os ecocardiogramas foram realizados com ecocardiógrafo ESAOTE-SIM 5000, com transdutores 2,5 e 3,5 MHZ, através dos modos unidimensional, bidimensional e Doppler, sendo obtida a massa ventricular esquerda pelo método de Devereux ${ }^{9}$ : massa $=1,04\left[(\mathrm{SIV}+\mathrm{VE}+\mathrm{PP})^{3}-\mathrm{VE}^{3}\right]-$ 13,6 , onde SIV = septo interventricular, $\mathrm{VE}=$ diâmetro diastólico interno do $\mathrm{VEe} \mathrm{PP}=$ parede posterior do $\mathrm{VE}$, tendo sido realizadas as medidas conforme padrão da Sociedade
Americana de Ecocardiografia ${ }^{10,11}$, e calculados os índices de massa do VE após a determinação da superfície corporal pela fórmula de Dubois ${ }^{12}: \mathrm{SC}=$ peso $^{0,427} \mathrm{X}$ altura ${ }^{0,718} \mathrm{X} 74,49$. Considerou-se como padrão ouro para HVE um índice de massa do $V E \geq 98 \mathrm{~g} / \mathrm{m}^{2}$ para mulheres e $\geq 120 \mathrm{~g} / \mathrm{m}^{2}$ para homens. Estes limites correspondem a valores de percentil de índice de massa maiores que $90 \%$ em uma amostra da população de 225 indivíduos saudáveis ${ }^{9}$.

O intervalo entre a realização dos exames (ECG e ecocardiograma) foi inferior a dois meses.

A análise estatística utilizou o teste normal ( $\mathrm{Z}$ ) de distribuição, para avaliação da proporção de concordância dos índices eletrocardiográficos em relação aos dados ecocardiográficos, sendo considerado como nível de significância estatística $\mathrm{p}<0,05$.

Foram calculados sensibilidade, especificidade, valores preditivos positivo e negativo, taxas de falso positivo e falso negativo e eficácia diagnóstica (capacidade do método para detectar resultados verdadeiros positivos ou negativos), para cada critério eletrocardiográfico isolado e para os quatro critérios considerados simultaneamente.

\section{Resultados}

Os valores obtidos para cada índice eletrocardiográfico encontram-se no quadro I, onde os pacientes estão enu-

\begin{tabular}{|c|c|c|c|c|c|c|c|c|c|c|c|c|c|}
\hline \multicolumn{14}{|c|}{ Quadro II - Dados obtidos ao ecocardiograma } \\
\hline $\mathrm{N}^{\circ}$ & Idade & Sexo & Cor & $\begin{array}{l}\text { Grau } \\
\text { HAS }\end{array}$ & $\begin{array}{l}\mathrm{AO} \\
\mathrm{mm}\end{array}$ & $\begin{array}{l}\mathrm{AE} \\
\mathrm{mm}\end{array}$ & $\begin{array}{c}\text { PPVE } \\
\text { mm }\end{array}$ & $\begin{array}{l}\text { SIV } \\
\mathrm{mm}\end{array}$ & $\begin{array}{l}\text { VED } \\
\mathrm{mm}\end{array}$ & $\begin{array}{c}\text { VES } \\
\mathrm{mm}\end{array}$ & $\begin{array}{c}\mathrm{FE} \\
\%\end{array}$ & $\begin{array}{c}\text { Massa } \\
\text { VEg }\end{array}$ & $\begin{array}{c}\text { IMVE } \\
\mathrm{g} / \mathrm{m}^{2}\end{array}$ \\
\hline 1 & 66 & M & $\mathrm{P}$ & $\mathrm{G}$ & 31 & 29 & 10 & 13 & 51 & 31 & 78 & 273 & 162 \\
\hline 2 & 61 & $\mathrm{~F}$ & B & M & 32 & 28 & 9 & 10 & 36 & 19 & 86 & 119 & 80 \\
\hline 3 & 33 & M & $\mathrm{P}$ & $\mathrm{L}$ & 34 & 38 & 10 & 10 & 53 & 34 & 74 & 240 & 117 \\
\hline 4 & 59 & M & B & $\mathrm{L}$ & 34 & 31 & 12 & 13 & 56 & 39 & 66 & 356 & 187 \\
\hline 5 & 72 & M & $\mathrm{P}$ & $\mathrm{G}$ & 31 & 37 & 12 & 13 & 44 & 32 & 62 & 243 & 139 \\
\hline 6 & 58 & $\mathrm{~F}$ & B & M & 34 & 31 & 13 & 13 & 34 & 19 & 83 & 177 & 97 \\
\hline 7 & 70 & $\mathrm{~F}$ & $\mathrm{P}$ & M & 32 & 28 & 10 & 14 & 42 & 28 & 70 & 213 & 118 \\
\hline 8 & 76 & $\mathrm{~F}$ & $\mathrm{P}$ & $\mathrm{L}$ & 31 & 32 & 10 & 11 & 46 & 26 & 83 & 202 & 113 \\
\hline 9 & 64 & $\mathrm{~F}$ & $\mathrm{~N}$ & $\mathrm{~L}$ & 34 & 34 & 13 & 13 & 54 & 26 & 89 & 355 & 186 \\
\hline 10 & 44 & $\mathrm{~F}$ & B & M & 31 & 39 & 10 & 11 & 50 & 28 & 82 & 231 & 136 \\
\hline 11 & 35 & M & $\mathrm{P}$ & $\mathrm{G}$ & 27 & 28 & 10 & 13 & 51 & 32 & 75 & 273 & 133 \\
\hline 12 & 60 & $\mathrm{~F}$ & B & $\mathrm{L}$ & 34 & 35 & 12 & 12 & 44 & 26 & 79 & 229 & 139 \\
\hline 13 & 26 & M & $\mathrm{N}$ & M & 35 & 37 & 13 & 15 & 53 & 32 & 78 & 383 & 190 \\
\hline 14 & 68 & $\mathrm{~F}$ & B & $\mathrm{L}$ & 31 & 32 & 9 & 10 & 41 & 22 & 85 & 147 & 94 \\
\hline 15 & 72 & M & B & $\mathrm{L}$ & 29 & 34 & 12 & 13 & 51 & 32 & 75 & 306 & 180 \\
\hline 16 & 61 & $\mathrm{~F}$ & B & M & 29 & 31 & 12 & 13 & 53 & 38 & 63 & 326 & 182 \\
\hline 17 & 53 & $\mathrm{~F}$ & $\mathrm{P}$ & $\mathrm{L}$ & 34 & 37 & 12 & 12 & 47 & 26 & 83 & 254 & 154 \\
\hline 18 & 56 & $\mathrm{~F}$ & $\mathrm{P}$ & $\mathrm{G}$ & 32 & 34 & 9 & 13 & 42 & 23 & 84 & 188 & 111 \\
\hline 19 & 72 & $\mathrm{~F}$ & B & $\mathrm{G}$ & 32 & 32 & 9 & 16 & 41 & 22 & 85 & 219 & 105 \\
\hline 20 & 49 & M & $\mathrm{P}$ & $\mathrm{G}$ & 38 & 34 & 12 & 13 & 53 & 34 & 74 & 326 & 176 \\
\hline 21 & 53 & $\mathrm{~F}$ & B & M & 37 & 31 & 10 & 12 & 51 & 29 & 82 & 256 & 148 \\
\hline 22 & 70 & $\mathrm{~F}$ & B & $\mathrm{G}$ & 34 & 38 & 11 & 14 & 48 & 32 & 70 & 304 & 171 \\
\hline 23 & 60 & M & B & $\mathrm{G}$ & 35 & 39 & 13 & 15 & 50 & 37 & 59 & 350 & 187 \\
\hline 24 & 59 & M & $\mathrm{P}$ & M & 34 & 32 & 13 & 13 & 49 & 33 & 70 & 303 & 167 \\
\hline 25 & 53 & $\mathrm{~F}$ & $\mathrm{P}$ & M & 33 & 28 & 12 & 13 & 50 & 37 & 59 & 297 & 176 \\
\hline 26 & 43 & $\mathrm{~F}$ & $\mathrm{P}$ & $\mathrm{L}$ & 29 & 32 & 10 & 12 & 50 & 32 & 74 & 248 & 137 \\
\hline 27 & 46 & $\mathrm{~F}$ & $\mathrm{P}$ & $\mathrm{L}$ & 28 & 32 & 9 & 10 & 48 & 28 & 80 & 190 & 110 \\
\hline 28 & 37 & $\mathrm{~F}$ & $\mathrm{P}$ & $\mathrm{L}$ & 34 & 22 & 9 & 9 & 41 & 22 & 85 & 136 & 88 \\
\hline 29 & 78 & $\mathrm{M}$ & B & $\mathrm{L}$ & 37 & 28 & 10 & 10 & 54 & 31 & 81 & 248 & 148 \\
\hline 30 & 61 & $\mathrm{M}$ & $\mathrm{P}$ & $\mathrm{L}$ & 38 & 29 & 15 & 15 & 48 & 23 & 89 & 364 & 184 \\
\hline
\end{tabular}




\begin{tabular}{|c|c|c|c|c|c|c|c|}
\hline \multicolumn{8}{|c|}{ Tabela I - Presença de um dos quatro critérios } \\
\hline Índices & SEN & ESP & VPP & VPN & PFP & PFN & EF DIAG \\
\hline Variáveis & $\%$ & $\%$ & $\%$ & $\%$ & $\%$ & $\%$ & $\%$ \\
\hline I - Sokolov & 40 & 100 & 100 & 25 & 0 & 75 & 50 \\
\hline II - Gubner & 28 & 80 & 46 & 19 & 54 & 81 & 37 \\
\hline III - Romhilt & 12 & 100 & 100 & 19 & 0 & 81 & 27 \\
\hline IV - Cornell & 12 & 100 & 100 & 19 & 0 & 81 & 27 \\
\hline
\end{tabular}

SEN- sensibilidade; ESP- especificidade; VPP- valor preditivo positivo; VPN- valor preditivo negativo; PFP- proporção de falsos positivos; PFN- proporção de falsos negativos; EF. DIAG- eficácia diagnóstica.

\begin{tabular}{|lccc|}
\hline \multicolumn{4}{|c|}{ Tabela II - Sensibilidade - comparação estatística } \\
\hline \multicolumn{1}{|c|}{ Índices } & $\mathrm{Z}$ & $\mathrm{P}$ & \multicolumn{1}{c|}{ Conclusão } \\
\hline Sokolov x Gubner & 1,00 & 0,15 & Diferença não significativa \\
Sokolov x Cornell e Romhilt & 2,55 & 0,0048 & Diferença significativa \\
Gubner x Cornell e Romhilt & 1,60 & 0,0495 & Diferença significativa \\
\hline
\end{tabular}

\begin{tabular}{|lccl|}
\hline \multicolumn{4}{|c|}{ Tabela III - Eficácia diagnóstica - comparação estatística } \\
\hline Índices & $\mathrm{Z}$ & $\mathrm{P}$ & Conclusão \\
\hline Sokolov x Gubner & 1,03 & 0,15 & Diferença não significativa \\
Sokolov x Cornell e Romhilt & 1,91 & 0,026 & Diferença significativa \\
Gubner x Cornell e Romhilt & 0,83 & 0,19 & Diferença não significativa \\
\hline
\end{tabular}

merados com seus respectivos dados (idade, sexo e cor) e classificados em hipertensos leves, moderados e graves.

Os dados obtidos ao ecocardiograma encontram-se no quadro II.

O índice de Sokolov mostrou sensibilidade de 40\%, especificidade de $100 \%$ e valor preditivo positivo de $100 \%$, sendo o mais eficaz na detecção da HVE, com eficácia diagnóstica de 50\%. A seguir, o índice de Gubner apresentou sensibilidade de $28 \%$, especificidade de $80 \%$ e eficácia diagnóstica de $37 \%$. Os índices de Romhilt e Cornell foram os de menor sensibilidade (12\%), com especificidade de $100 \%$ e eficácia diagnóstica de $27 \%$ (tab. I).

Não houve diferença significativa em relação à sensibilidade dos índices de Sokolov e Gubner (40\% vs 28\%; $\mathrm{p}=0,15$ ), porém a diferença foi significativa quando comparado Sokolov com Cornell ou Romhilt (40\% vs 12\%; $\mathrm{p}=0,0048)$ e Gubner com Cornell ou Romhilt ( $28 \%$ vs $12 \%$; $\mathrm{p}=0,0495)$ (tab. II).

Quanto à eficácia diagnóstica, a diferença foi significativa quando comparados os índices de Sokolov e Cornell ou Romhilt ( $50 \%$ vs $27 \%$; $\mathrm{p}=0,026$ ), porém não houve significância estatística na diferença entre os índices de Sokolove
Gubner (50\% vs 37\%; p=0,15), e Cornell ou Romhilt e Gubner $(27 \%$ vs $37 \%$; $\mathrm{p}=0,19)$ (tab. III).

Quando considerados os quatro índices eletrocardiográficos simultaneamente, foram encontrados sensibilidade de $52 \%$, especificidade de $80 \%$, valor positivo de $93 \%$ e eficácia diagnóstica de 57\% (tab. IV).

\section{Discussão}

Os dados da literatura mostram valores controversos em relação à sensibilidade e especificidade dos índices eletrocardiográficos na detecção de HVE. Discrepâncias que nos levam a questionar a valorização de tais critérios no diagnóstico de HVE. Assim, passamos a perguntar qual o verdadeiro significado dos achados do ECG em relação às alterações anátomo-fisiológicas cardíacas. Os critérios de sobrecarga ou HVE significariam hipertrofia miocárdica com espessamento do miocárdio (miócitos dispostos em paralelo) ou estados de sobrecarga pressórica ou volumétrica com dilatação ventricular (miócitos dispostos em série), sem espessamento miocárdico significativo?

Observou-se que no subgrupo com HAS moderada ou grave (17 dos 30 pacientes), sete ( $41 \%$ ) não apresentavam HVE ou era discreta ao ecocardiograma. Desses pacientes, todos referiram diagnóstico de HAS há mais de um ano e tratamento regular durante o período. Dentre os demais pacientes (10 com HVE moderada ou importante), nove tiveram diagnóstico conhecido de HAS há mais de um ano e um diagnóstico recente, cinco referiram tratamento irregular, dois uso regular de metildopa, um uso regular de captopril, nitrendipina e furosemida, um uso regular de hidroclortiazida e um sem tratamento prévio.

Estes resultados sugerem uma nítida correlação entre a menor incidência de HVE e o tratamento regular da HAS. Porém, não foi observada relação entre a gravidade da HVE e o tempo de início da doença, uma vez que, nesse grupo de pacientes, apenas um desconhecia o diagnóstico e os de-

\begin{tabular}{|ccccccc|}
\hline \multicolumn{5}{|c|}{ Tabela IV - Presença de algum (pelo menos um) dos quatro índices considerados simultaneamente } \\
\hline SEN \% & ESP \% & VPP $\%$ & VPN \% & PFP \% & PFN \% & EF. DIAG \% \\
\hline 52 & 80 & 93 & 27 & 7 & 73 & 57 \\
\hline $\begin{array}{l}\text { SEN- sensibilidade; ESP- especificidade; VPP- valor preditivo positivo; } \\
\text { de falsos negativos; EF. DIAG- eficácia diagnóstica. }\end{array}$ & & & & & \\
\hline
\end{tabular}


mais tinham diagnóstico conhecido há mais de um ano. Também não foram observadas diferenças significativas quanto à gravidade da HVE em relação a sexo ou raça.

O presente trabalho, com este pequeno grupo de pacientes, encontrou resultados de sensibilidade e especificidade para os critérios eletrocardiográficos, discordantes dos da literatura no que diz respeito ao melhor critério, porém, houve concordância em relação à baixa sensibilidade do ECG. Casale e col encontraram sensibilidade de $22 \%$ e especificidade de $100 \%$ para o índice de Sokolov-Lyon, $33 \%$ de sensibilidade e $94 \%$ de especificidade para o índice de Romhilt, e $42 \%$ de sensibilidade e $96 \%$ de especificidade para o índice de Cornell ${ }^{8}$.

Romhilt e col encontraram sensibilidade de 56,3\% e especificidade de $12,5 \%$ para o índice de Sokolov, e 53,8\% de sensibilidade para o índice de Romhilt ${ }^{4}$. Clemency e col apontaram sensibilidade e especificidade maiores do que $70 \%$, considerando o índice de Sokolov positivo se maior que $30 \mathrm{~mm}$ e o índice de Romhilt $\geq 4$ pontos ${ }^{5}$.

Nossos resultados indicaram o índice de Sokolov, com sensibilidade de $40 \%$ e especificidade de $100 \%$, como o mais eficaz na detecção da HVE. O índice de Gubner apresentou sensibilidade de $28 \%$ e especificidade de $80 \%$, sendo que não houve diferença estatisticamente significativa entre este índice e o índice de Sokolov em relação à sensibilidade $(\mathrm{p}=0,15)$. Os índices de Cornell e Romhilt apresentaram sensibilidade de $12 \%$ e especificidade de $100 \%$, havendo diferença estatisticamente significativa em relação à sensibilidade do índice de Sokolov ( $\mathrm{p}=0,0048)$. Quando comparadas as sensibilidades dos índices de Gubner, Cornell e Romhilt, a diferença foi significativa $(\mathrm{p}=0,0495)$.

Quanto à eficácia diagnóstica dos critérios eletrocardiográficos analisados, o índice de Sokolov (o mais eficiente) não foi superior ao segundo colocado - o índice de Gubner -, porém sua eficácia foi significativamente melhor quando comparada aos índices de Cornell ou Romhilt $(\mathrm{p}=0,026)$. Comparando o índice de Gubner com Cornell ou Romhilt não houve diferença estatisticamente significativa.

Quando considerados os quatro critérios de ECG, simultaneamente, a sensibilidade foi de $52 \%$ e especificidade de $80 \%$, com eficácia diagnóstica de $57 \%$, ou seja, houve melhora discreta em relação ao melhor índice individual (Sokolov com $40 \%$ de sensibilidade).

Nossos resultados demonstraram que os métodos eletrocardiográficos, considerados individualmente ou em conjunto, foram pouco eficazes na detecção da HVE. Mesmo num subgrupo de 12 pacientes de nossa amostra, com HVE mais importante (índice de massa do $\mathrm{VE} \geq 160 \mathrm{~g} / \mathrm{m}^{2}$ ), considerando os quatro critérios simultaneamente, a sensibilidade atingiu somente $50 \%$, ratificando a baixa eficácia do método eletrocardiográfico no diagnóstico de HVE, embora seja este método o mais acessível na rotina diagnóstica cardiovascular.

Frente à grande importância da HVE como fator de risco independente na morbimortalidade cardiovascular ${ }^{2}$, concluímos que atualmente impõe-se a utilização de método propedêutico mais sensível na detecção desta entidade patológica, sobretudo em fases mais precoces.

\section{Referências}

1. Kannel WB, Gordon T, Offutt D - Left Ventricular Hypertrophy by Electrocardiogram - Prevalence, Incidence and Mortality in the Framingham Study. Ann Intern Med 1969; 71: 89-101.

2. Bestetti RB - Aspectos epidemiológicos da hipertrofia ventricular esquerda. Rev Soc Cardiol Est SP 1994; 4: 313-9.

3. Farb A, Devereux RB, Kligfield P - Day-to-day variability of voltage measurements used in electrocardiographic criteria for left ventricular hypertrophy. J Am Coll Cardiol 1990; 15: 618-23.

4. Carneiro EF - O Eletrocardiograma 10 Anos Depois. Rio de Janeiro: Enéas Ferreira Carneiro, 1992: 119-39.

5. Arroyo JB, Braga JMS, Lina Filho B, Pfeferman A - Análise crítica do eletrocardiograma e do vetorcardiograma no diagnóstico da hipertrofia ventricular esquerda. Rev Soc Cardiol Est SP 1994; 4: 353-60.

6. The Fifth Report of the Joint National Committee on Detection, Evaluation and Treatment of High Blood Pressure - JNC(V). Arch Intern Med. 1993; 153: 154-83.

7. Gubner R, Ungerleider HE - Electrocardiographic criteria of left ventricular hypertrophy. Arch Intern Med 1943; 72: 196-209.
8. Casale PN, Devereux RB, Alonso DR, Campo E, Kligfield P - Improved sexspecific criteria of left ventricular Hypetrophy for clinical and computer interpretation of electrocardiograms: validation with autopsy findings. Circulation 1987; 75: 565-72.

9. Lièvre $\mathrm{M}$, Guéret $\mathrm{P}$, Gayet $\mathrm{C}$ et al - Ramipril - induced regression of left ventricular hypertrophy in treated hypertensive individuals. Hypertension 1995; 25: 92-7.

10. Henry WL, De Maria A, Gramiak R et al - Report of the American Society of Echocardiography Committee on Nomenclature and Standards in Twodimensional Echocardiography. Circulation 1980; 62: 212-8.

11. Sahn DJ, De Maria A, Kisslo J, Weyman A - The Committee on M- Mode Standardization of the American Society of Echocardiography. Recommendations Regarding Quantitation in M- Mode Echocardiography: Results of a Survey of Echocardiographic Measurements. Circulation 1978; 58: $1072-83$.

12. Rippe JM, Irwin RS, Alpert JS, Fink MP - Intensive Care Medicine. $2^{\text {nd }}$ ed. Boston: Little, Brown Co, 1991: 2005. 